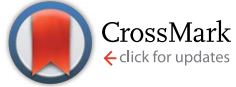

Cite this: RSC Adv., 2016, 6, 83399

\title{
New strategy for magnetic gas sensing
}

R. Ciprian, ${ }^{\text {*a }}$ P. Torelli, ${ }^{\text {b } A . ~ G i g l i a, ~}{ }^{\text {b B. Gobaut }}{ }^{\text {a }}$ B. Ressel, ${ }^{c}$ G. Vinai, ${ }^{b}$ M. Stupar, ${ }^{c}$ A. Caretta, ${ }^{a}$ G. De Ninno, ${ }^{\text {ac }}$ T. Pincelli, ${ }^{\text {bd }}$ B. Casarin, ${ }^{\text {ae }}$ G. Adhikary, ${ }^{c}$ G. Sberveglieri, ${ }^{f}$ C. Baratto ${ }^{f}$ and M. Malvestuto ${ }^{a}$

A new-concept approach to room temperature magnetic gas sensing has been developed, based on newly designed $\mathrm{Co} / \mathrm{ZnO}$ hybrid nanostructures. The sensor prototype has been demonstrated to be sensitive, reversible, fast and scalable. In this work, the role of the $\mathrm{Co} / \mathrm{ZnO}$ surface and interface in the gas-sensing mechanism has been clarified. In order to attain a comprehensive understanding of the physics governing the remarkable properties of the $\mathrm{Co} / \mathrm{ZnO}$ stack, an extensive electronic and structural investigation has been carried out. The reaction at room temperature with target gases involves the surface and the lateral faces of the $\mathrm{ZnO}$ nanorods, with the formation of structural defects and vacancies. In particular, it has been discovered that the stress enhancement as well as the change in the polarizability of the ZnO nanorods are transduced by $\mathrm{Co}$ in a change of its magnetization. The interplay between these phenomena may provide versatile approaches to tune the intrinsic electronic, magnetic and optical properties of the hybrid nanostructure.
Received 17th July 2016

Accepted 28th August 2016

DOI: 10.1039/c6ra18213e

www.rsc.org/advances magnetic properties. In addition, the use of intense external magnetic field sources must be replaced with alternative solutions, in order to ensure the device portability and scalability.

As a matter of fact, nowadays the related research activity is addressed to exploit dilute magnetic semiconductors ${ }^{3}$ and/or transition metal oxides, ${ }^{5}$ both requiring the application of very large magnetic fields and, often, to work at low temperatures, characteristics that hinder the scalability and portability of the devices.

In order to overcome these limits, Matatagui et al. ${ }^{6}$ proposed an innovative, simple and inexpensive magnonic gas sensor constitutes by $\mathrm{CuFe}_{2} \mathrm{O}_{4}$ magnetic nanoparticles deposited on top of a magnetostatic spin wave tunable oscillator of YIG. The huge sensitivity and reproducibility, as well as the low response time of the developed sensor pave the way towards the development of innovative and challenging new magnetic devices. Indeed, very recently a very simple and low-cost system has been developed based on a hybrid ferromagnet/piezoelectric-semiconductive oxide, 7 constituted by a continuous Co layer capped by $\mathrm{ZnO}$ nanorods (NRs), magnetoelectrically coupled at the interface. A controllable and scalable preparation procedure has been optimized, making use of a single deposition technique, the radiofrequency (RF) magnetron sputtering. The prototype has been demonstrated to be a stable, sensitive and fast magnetic gas sensor, working efficiently at room temperature (RT) and with very small applied magnetic fields ( $\approx 50 \mathrm{Oe})$. On the base of the obtained results, a model for the magnetic gas sensing phenomenon is presented.

The obtained results not only shed light on the magnetic gas sensing phenomenology, but also contribute to tackle the puzzle of the outstanding properties of hybrid magnetoelectric systems. 


\section{B. Experimental section}

The system has been fabricated with a RF magnetron sputtering system on polycrystalline $2 \times 2 \mathrm{~mm}^{2} \mathrm{Al}_{2} \mathrm{O}_{3}$ substrates. A Co layer, $100 \mathrm{~nm}$ thick, has been directly deposited onto the substrates at RT. At $400{ }^{\circ} \mathrm{C}$, a very thin and non-continuous Sn layer (a few angstroms thick) has been deposited. Sn acts as a catalyst for the subsequent growth of the ZnO NRs. The NRs have been fabricated, via a self-assembly process, with a RF power of $150 \mathrm{~W}$ at $10^{-2}$ mbar for different growing times. ${ }^{7,8}$

We prepared two kinds of samples: sample MGS1, with a growing time fixed to $90 \mathrm{~s}$ in order to study the early stage of NRs formation; sample MGS2, for which the deposition time was $300 \mathrm{~s}$, characterized by the complete NRs formation. In order to stabilize the $\mathrm{ZnO}$ and to enrich it of oxygen, the samples have been annealed in air at $300{ }^{\circ} \mathrm{C}$ for $1 \mathrm{~h}$; the two annealed samples have been called MGS1A and MGS2A.

The morphology has been analyzed by field-emission scanning electron microscopy (FE-SEM, Zeiss Supra 40). X-ray diffraction data indicate that Co grew in its hep structure, while $\mathrm{ZnO}$ shows a hexagonal wurtzite structure. ${ }^{7}$

The magnetic properties have been analyzed at RT using a magneto-optical Kerr effect (MOKE) magnetometer in longitudinal geometry, using an s-polarized He-Ne laser (633 nm) and the intensity modulation technique at frequency of $415 \mathrm{~Hz}$. The experimental setup is schematically depicted in Fig. 1. This technique was exploited for the magnetic gas sensing measurements, placing the samples and the coils inside a stainless steel chamber (volume: $1 \mathrm{~L}$ ) with gas inlet and outlet. The measurements were carried out at RT in a constant flow of humid synthetic air ( $\mathrm{RH})$ at $50 \%$, recording the change in the magnetization due to the target gas insertion in the chamber.

XPS measurements were acquired using a Scienta R3000 hemispherical analyzer and a monochromatic $\mathrm{Al} \mathrm{K}_{\alpha}(1486.6 \mathrm{eV})$ $\mathrm{X}$-ray source. For the quantitative analysis of the $\mathrm{Zn} 2 \mathrm{p}$ and $\mathrm{O} 1 \mathrm{~s}$ spectral features, the raw data were fitted with Voigt function.

NEXAFS and XMCD at the Co and $\mathrm{Zn} \mathrm{L}_{2,3}$ edges, and $\mathrm{O}$ K edge have been collected at RT at BEAR Beamlines of the Elettra synchrotron (Trieste, Italy). ${ }^{9}$ The spectra have been acquired in total electron and fluorescence yield mode, TEY and TFY

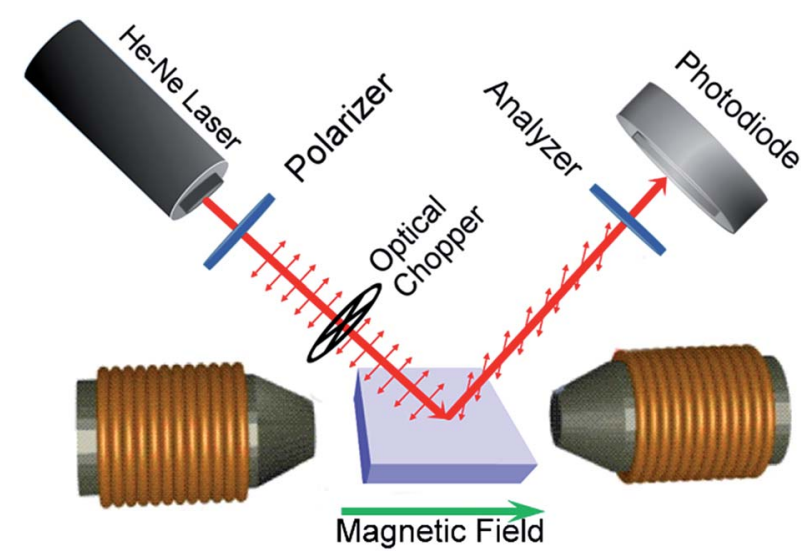

Fig. 1 Schematic view of the MOKE experimental setup. respectively, and normalized to the incident photon flux $I_{0}$, measured on a grid placed at the experimental chamber. In order to allow a direct comparison of the spectral features, all the spectra were normalized to the intensity of the most intense peak. The light was circularly polarized with $70 \%$ of polarization degree, and the beam was impinging at $45^{\circ}$ with respect to the surface normal.

The XMCD spectra were measured on samples in their remanence state, after applying an in-plane magnetic field pulse of about 250 Oe. The XMCD signal was evaluated as the difference between the spectra acquired applying opposite directions of the magnetic field pulse and normalized to the intensity of the most intense NEXAFS peak.

\section{Results}

The ZnO NRs/Co device is schematically depicted in Fig. 2a together with a SEM cross-section image. An irregular and continuous layer of $\mathrm{ZnO}$ forms on the Co layer, characterized by a columnar structure. These columns represent the seeds for the nanorods self-assembly.

Two samples were carefully analyzed, constituted by $\mathrm{ZnO}$ NRs with different lengths, as outlined in the Experimental section. Sample called MGS1, with a ZnO-NRs deposition time of $90 \mathrm{~s}$, shows NRs in the early stage of their formation, and sample referred as MGS2 characterized by completely formed NRs, indeed the deposition time was set to $300 \mathrm{~s}$.

Sample MGS1 is characterized by short NRs (100-200 nm long) with irregular tips (Fig. 2b). Sample MGS2 shows a better crystallinity, with NRs $\sim 1 \mu \mathrm{m}$ long and average diameter of 20-30 nm (Fig. 2c). SEM and XRD analysis (not shown) indicate that the morphology and crystallinity of both samples do not change as a consequence of the thermal treatment (performed in air at $300^{\circ}$ for $1 \mathrm{~h}$ ), a standard procedure to stabilize and enrich in oxygen the ZnO NRs. ${ }^{10}$ After annealing, the samples are called respectively MGS1A and MGS2A.

Fig. 3 shows the O K-edge NEXAFS spectra recorded in total fluorescence and electron yield mode for the samples before
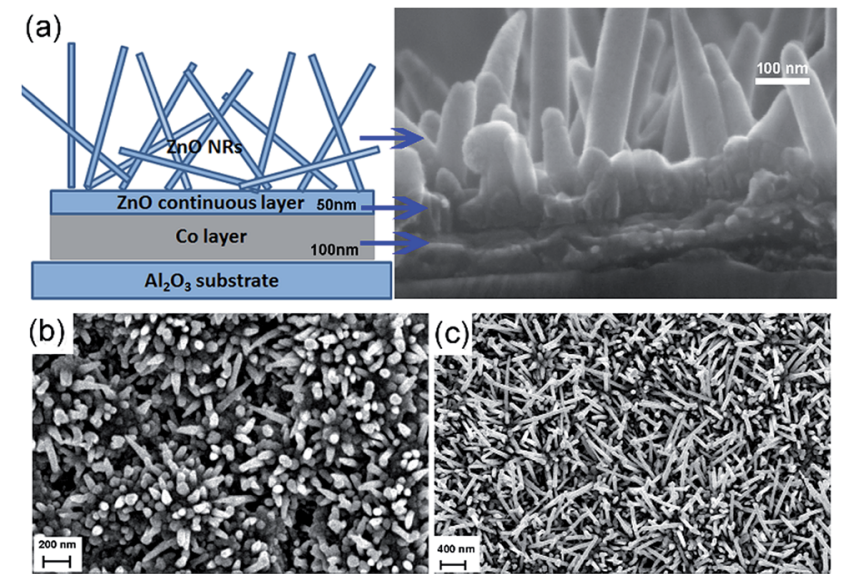

Fig. 2 (a) Schematic picture of the ZnO NRs/Co system and a SEM cross-section image of MGS1. Morphology of (b) MGS1 and (c) MGS2. 
(MGS1 and MGS2) and after (MGS1A and MGS2A) the annealing. The absorption cross section at the different photon energies selectively probes the $\mathrm{O}-\mathrm{Zn}$ and $\mathrm{O}-\mathrm{Co}$ bonds, providing an insight on oxygen orbital topology. The $\mathrm{O} \mathrm{K}$ edge displays two main features $\mathrm{A}_{1}-\mathrm{A}_{2}$ in the $530-539 \mathrm{eV}$ range, which stem from the hybridization of $\mathrm{O} 2 \mathrm{p}-\mathrm{Zn} 4 \mathrm{~s} / \mathrm{Co} 3 \mathrm{~d}$ states.

The $\mathrm{A}_{3}-\mathrm{A}_{4}$ features in the $539-550 \mathrm{eV}$ range can be ascribed to the hybridization of $\mathrm{O} 2 \mathrm{p}-\mathrm{Zn} 4 \mathrm{p}$ and Co $4 \mathrm{sp}$ states, while $\mathrm{A}_{5}-\mathrm{A}_{7}$, above $550 \mathrm{eV}$, are assigned to $\mathrm{O} 2 \mathrm{p}-\mathrm{Zn} 4 \mathrm{~d}$ states. ${ }^{11}$ The $\mathrm{A}_{1}$ pre-edge peak is the most sensitive spectral feature to the formation and amount of $\mathrm{O}$ vacancies $\left(\mathrm{V}_{\mathrm{O}}\right)$ as well as to the diffusion of Co into $\mathrm{ZnO}$ which induces unoccupied $3 \mathrm{~d}$ states inside the ZnO energy band gap. The NRs stretching and the improved crystallinity of MGS2 in comparison with MGS1, give rise to a significant increase of the relative intensity of the $A_{1}$ peak with respect to $A_{2}$ (Fig. 3a). This is due to an increased amount of $\mathrm{V}_{\mathrm{O}},{ }^{12,13}$ since the stretching of the NRs cannot change the amount of Co diffusing into $\mathrm{ZnO}$. The increase in the $\mathrm{V}_{\mathrm{O}}$ amount can be explained considering the co-sputtering process. The longer deposition time for MGS2-ZnO NRs may well dictate a decrease in the $\mathrm{O}$ concentration of the $\mathrm{ZnO}$-sputtering target during the deposition. The origin of this resides in the different growing rates of $\mathrm{O}$ and $\mathrm{Zn}$ during the co-deposition, causing the formation of an off-stoichiometric compound.

This interpretation is corroborated by the $\mathrm{A}_{1}$ and $\mathrm{A}_{2}$ relative intensities in TEY spectra (Fig. $3 \mathrm{~b}$ and c), which only probe the

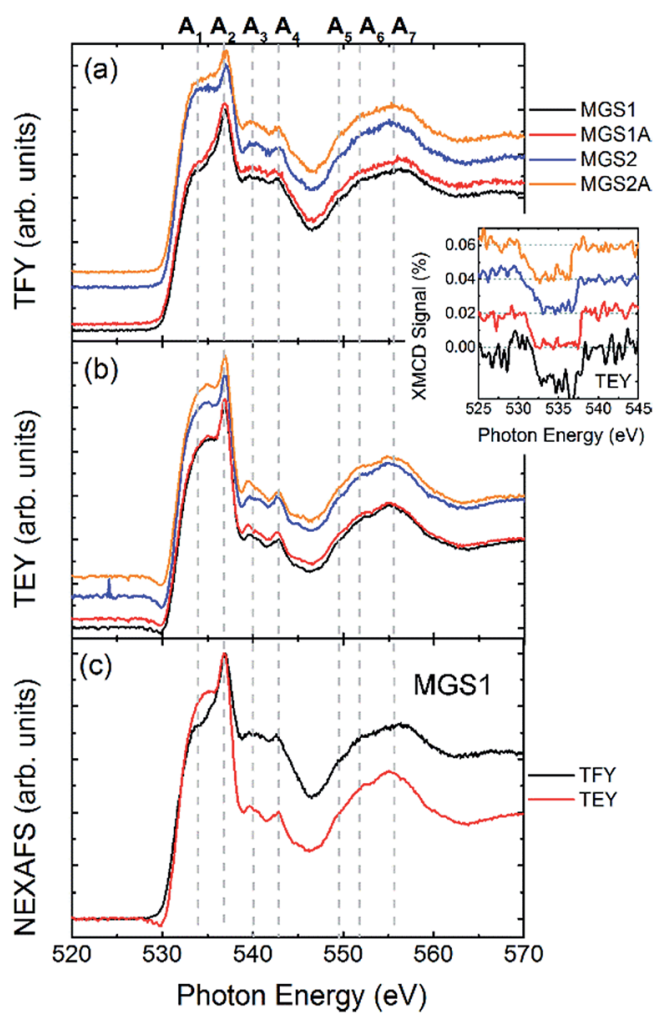

Fig. 3 NEXAFS spectra at O K edge acquired in (a) TFY and (b) TEY for samples MGS1 and MGS2 before and after the annealing in air (MGS1A and MGS2A). Inset: TEY-XMCD signal. The curves have been shifted along the vertical scale for sake of clarity. (c) Comparison between the TEY and TFY spectra acquired for MGS1 in the as grown state. surface and edge layers of the $\mathrm{ZnO}$ NRs. The huge increase (almost 2 times) in the relative intensity of $\mathrm{A}_{2}$ with respect to that of $\mathrm{A}_{3}$ and $\mathrm{A}_{4}$ contributions (Fig. 3c) reflects a rise in the structural disorder, due to the formation of structural defects and $\mathrm{V}_{\mathrm{O}}$ in the surface/edge layers. ${ }^{13}$ The absence of pre-edge features at energies lower than $530 \mathrm{eV}$ in both TFY and TEY spectra, which are normally ascribed to transition from $\mathrm{O} 1 \mathrm{~s}$ to O 2p states hybridized with the unoccupied states of the transition metal 3d state, ${ }^{\mathbf{1 4}}$ suggests that the Co diffusion into $\mathrm{ZnO}$ is quite limited to a very thin interfacial region. The NRs of both samples are almost unaffected by the thermal treatment.

We carefully analyzed the magnetic contrast at the $\mathrm{O} K$ edge. A very small magnetic moment has been found on $\mathrm{O}$ atoms in the TEY spectra (inset of Fig. 3), ${ }^{15,16}$ while almost no contrast was detected in TFY spectra. This magnetic contrast at $\mathrm{O} K$ edge is not affected by either the increase in length or the annealing of the NRs.

The absence of a magnetic contrast in TFY spectra implies that the main contribution arises from the surface and lateral faces of the $\mathrm{ZnO} \mathrm{NRs}$. It is reasonable to assume that an important role is played by oxygen vacancies and structural disorder, both increasing in proximity of the NRs surfaces. These findings are in agreement with the work of Singh et al. ${ }^{\mathbf{1 5}}$ on ZnO nanostructures which demonstrated both theoretically and experimentally that magnetism in pure $\mathrm{ZnO}$ is almost due to defects, vacancies or dangling/unpaired $2 \mathrm{p}$ bonds at $\mathrm{O}$ sites around $\mathrm{Zn}$ vacancy $\left(\mathrm{V}_{\mathrm{Zn}}\right)$ centers, which are primary located in the impurity or surface/edge regions, rather than in the core of the nanostructures.

The features in the $\mathrm{Zn} \mathrm{L}_{2,3}$ spectra measured in TFY and TEY (not shown) are due to transition from $2 \mathrm{p}$ to $4 \mathrm{~d} / \mathrm{s}$ states of $\mathrm{Zn},{ }^{17,18}$ since the $3 \mathrm{~d}$ orbital of $\mathrm{Zn}$ is fully occupied. The spectra are completely unaffected by both the NRs lengthening and the sample annealing, moreover no magnetic moment at the $\mathrm{Zn} 4 \mathrm{~d}$ states was detected.

Fig. 4 shows the TFY spectra at Co $\mathrm{L}_{2,3}$ edges. Screening effects of the ZnO NRs have prevented the detection of the TEY Co signal, confirming that Co only diffuses into a very thin $\mathrm{ZnO}$ NRs/Co interfacial layer, leaving the nanostructures free from Co contaminations. Conversely, the TFY spectra originate from metallic Co. The combination of the thermal treatment and the NRs stretching induces an enhancement of the spectral features indicated by arrows in Fig. 4 and ascribed to the presence of Co oxides. The absence of a pre-edge $\mathrm{L}_{3}$ peak and the shape itself of the $\mathrm{L}_{3}$ peak suggest that these localized states are mainly due to Co $3+$ valence. ${ }^{19,20}$

Sample MGS1 shows a clear magnetic contrast, which at the $\mathrm{L}_{3}$ edge reaches the $8 \%$ and remains unchanged after the annealing treatment (Fig. 4 - bottom panel). The absence of magnetic contrast for sample MGS2 and MGS2A can only be ascribed to the measurements, which were carried out at sample remanence. Indeed, while MGS1 is characterized by a well square-shaped hysteresis loop, the NRs stretching gives rise to a significant shearing of the loop (Fig. 4 - inset). Moreover, the MGS2 loop is not completely closed at the highestapplicable magnetic field suggesting that it represents only a minor loop. 


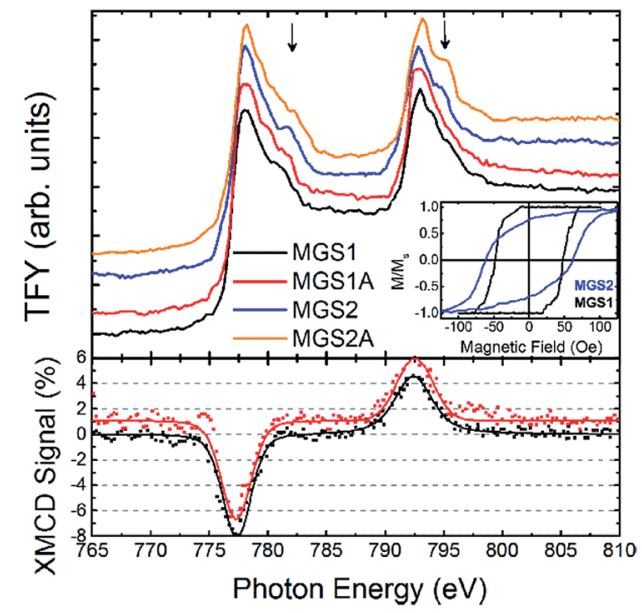

Fig. 4 NEXAFS spectra acquired in TFY at $C_{0} L_{2,3}$ edges and the corresponding XMCD signals. The spectra have been shifted along the vertical scale only for sake of clarity. The hysteresis loops of MGS1 and MGS2 are shown in the inset.

Thanks to the good and suitable morphology and structure, MGS2A was extensively used for two months for the detection of different gases $\left(\mathrm{C}_{3} \mathrm{H}_{6} \mathrm{O}, \mathrm{CO}\right.$ and $\left.\mathrm{H}_{2}\right)$ at different concentrations. The magnetic measurements were performed at RT using a magneto-optical Kerr effect magnetometer, which measures the change of the polarization of a linearly polarized light after reflection on a magnetized sample surface (Fig. 1). For the magnetic gas sensing measurements, the applied field was kept constant to 50 Oe, while the signal collected by a Si photodiode was continuously monitored. The results are summarized in Fig. 5 (black line). The sample annealing has been demonstrated to be fundamental in order to stabilize the recorded magnetic signal and to increase the signal-to-noise ratio.

The sensor shows good sensitivity to all the tested gases even at the lowest concentration, with the highest sensitivity towards hydrogen. Moreover the signal increases almost linearly with increasing concentration of all tested target gases. The detection limits defined as the value of sensor sensitivity greater than three times the standard deviation of the background noise signal are 10, 5 and 4 ppm for $\mathrm{C}_{3} \mathrm{H}_{6} \mathrm{O}, \mathrm{CO}$ and $\mathrm{H}_{2}$ respectively. The stability is quite good with the complete recovery of the baseline after gas exposure; the response and recovery time are less than $60 \mathrm{~s}$. The experiment was repeated using sample MGS2A in a zero applied magnetic field (Fig. 5 - blue line). In this configuration, the recorded signal is sensitive only to changes in the sample reflectivity, possibly occurring as a consequence of the reaction with the target gases. The results (i.e. zero signal for all concentrations and gases) underline the magnetic nature of the sensor response, being unaffected, during the reaction, to changes of either the reflectivity or the optical properties of the $\mathrm{ZnO}$ NRs.

The gas sensing tests were also carried out using, as sensing material, a layer of pure Co (100 nm thick) and the MGS1A sample, characterised by a quite different morphology with respect to that of MGS2A (Fig. 2b and c). The obtained results

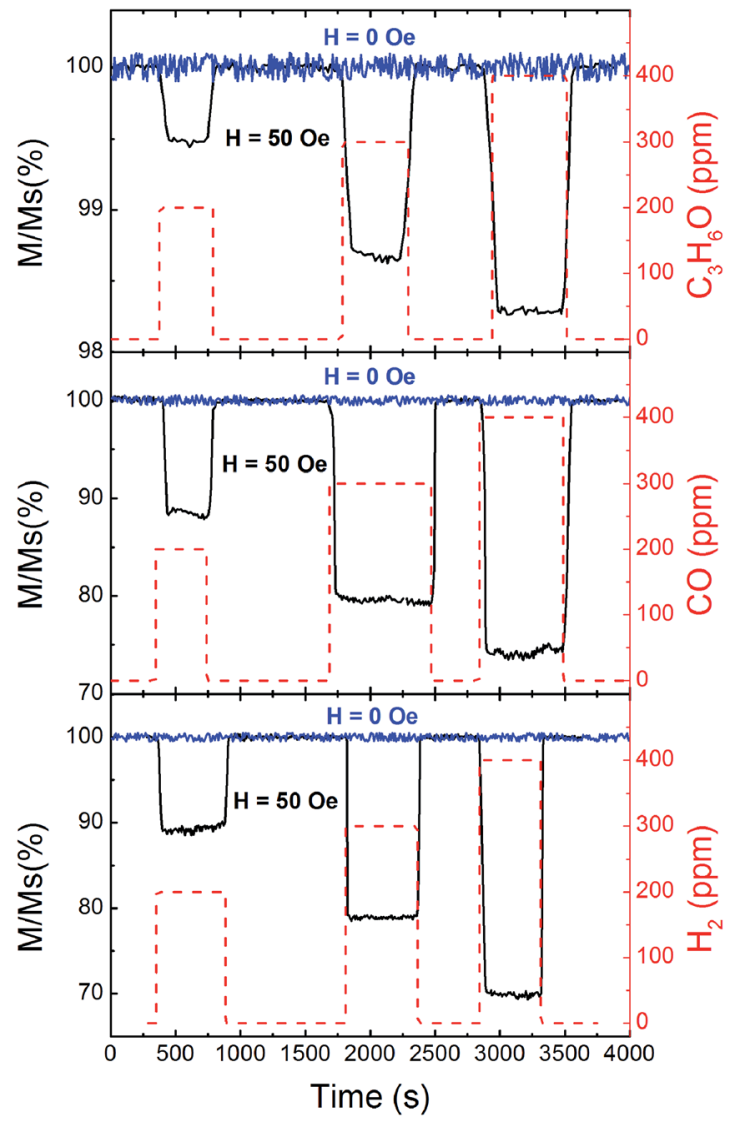

Fig. 5 RT changes of the MGS2A magnetization induced by its exposure and reaction with different concentrations of $\mathrm{C}_{3} \mathrm{H}_{6} \mathrm{O}, \mathrm{CO}$ and $\mathrm{H}_{2}$ target gases with (50 $\mathrm{Oe}$ - black line) and without (blue line) an applied magnetic field.

allow a better understanding of the role played by the different constituents of the system during gas detection and reaction.

The signal recorded during $\mathrm{H}_{2}$ injection, using a film of pure Co, quickly decreased down to the complete disappearance. The humid atmosphere $(\mathrm{RH}=50 \%)$ combined with the fast reactivity and oxidation of Co gave rise to a change in the sample reflectivity and to film exfoliation.

The comparison of the performances of MGS1A and MGS2A, emphasizes the role of the $\mathrm{ZnO}$ nanorods in the magnetic gas sensing process. Fig. 6 shows the results obtained using the two

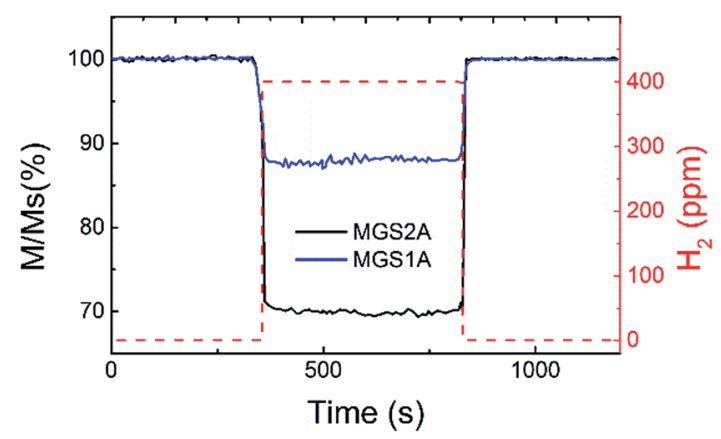

Fig. 6 Comparison of the RT magnetic gas sensing performance of samples MGS1A and MGS2A exposed to 400 ppm of $\mathrm{H}_{2}$. 

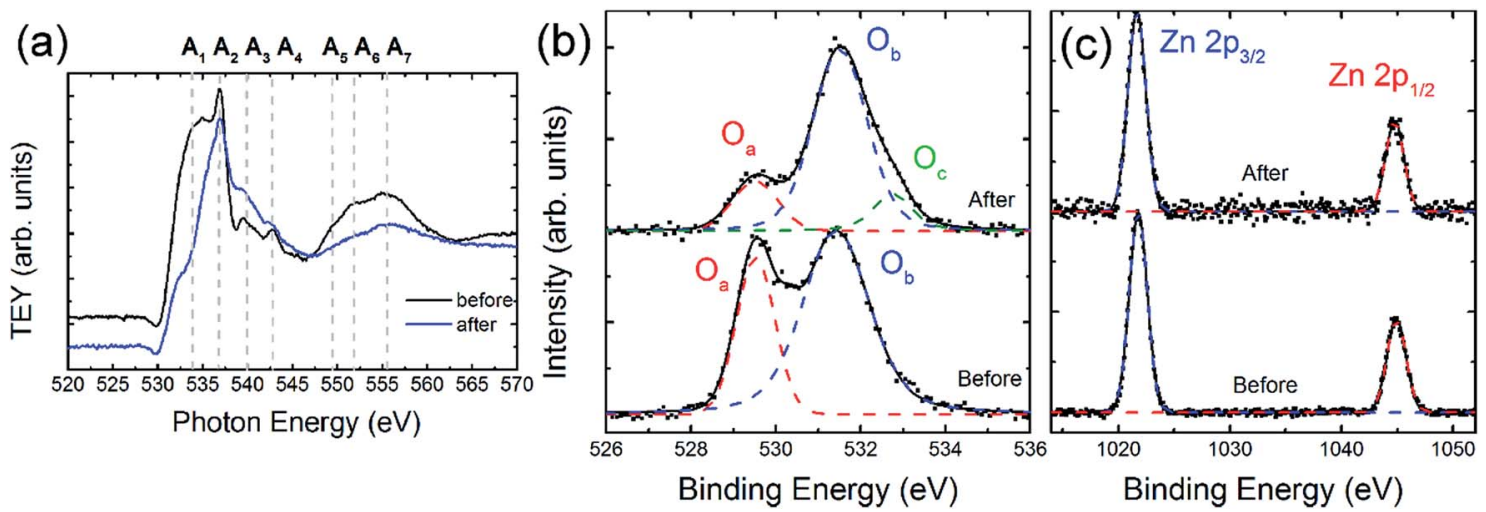

Fig. 7 (a) Comparison of the TEY-NEXAFS spectra at $O \mathrm{~K}$ edge, deconvoluted (b) $\mathrm{O}$ 1s and (c) Zn 2p XPS core level spectra of MGS2A, before and after gas exposure to $\mathrm{H}_{2}$ target gas (400 ppm).

samples and $\mathrm{H}_{2}$ (400 ppm) as target gas. The signal recorded for sample MGS1A is a factor two lower with respect to that obtained considering sample MGS2A.

Longer nanorods and better crystallinity, characterising sample MGS2A, are improving the room temperature $\mathrm{ZnO}$ reactivity towards the target gas. These results indirectly suggest the importance of the $\mathrm{ZnO}$ nanostructuration in the gas sensing mechanism: the increase of the surface to volume ratio is the key parameter in order to improve and enhance the chemical sensitivity of the sensor.

NEXAFS measurements were performed on MGS2A after reaction with $\mathrm{H}_{2}$ target gas. The data indicate that gas detection is purely a surface phenomenon, which mainly involves oxygen specie. Indeed, no changes were detected in the TFY and TEY Zn and Co $\mathrm{L}_{2,3}$ spectra as well as in the TFY O K spectra. On the contrary, the TEY spectra at the $\mathrm{O} \mathrm{K}$ edge (Fig. 7a) drastically change after gas exposure. The $\mathrm{A}_{1}$ peak drops, while the $\mathrm{A}_{3}$ and $\mathrm{A}_{4}$ features are less defined and broad, with a concomitant increase of their intensities with respect to that of $\mathrm{A}_{2}$ peak. These significant changes of the TEY spectral features indicate an increased amount of surface structural and interstitial $\mathrm{Zn}$ defects inducing the $\mathrm{O} 2 \mathrm{p}$ states to be more dispersed.

In order to better understand this finding and to further investigate the oxidation states of the sample surface, XPS survey spectra and high resolution scan on $\mathrm{Zn} 2 \mathrm{p}$ and $\mathrm{O} 1$ s were performed. All the peaks of the wide range XPS spectrum (not shown) can be attributed to $\mathrm{Zn}, \mathrm{O}$ and $\mathrm{C}$ species. The carbon contamination is not surprising, considering that the device works in air. The high resolution scan for MGS2A before and after RT magnetic gas sensing are shown in Fig. $7 \mathrm{~b}$ and c, respectively, for $\mathrm{O} 1 \mathrm{~s}$ and $\mathrm{Zn} 2 \mathrm{p}$. The $\mathrm{O} 1 \mathrm{~s}$ spectra (Fig. $7 \mathrm{~b}$ bottom) can be deconvoluted into two main components. The $\mathrm{O}_{\mathrm{a}}$ peak, located at $529.4 \mathrm{eV}$, corresponds to lattice $\mathrm{O}^{2-}$ in $\mathrm{ZnO}$ crystal and the $\mathrm{O}_{\mathrm{b}}$ peak, at $531.5 \mathrm{eV}$, to $\mathrm{O}^{2-}$ in oxygen deficient regions. ${ }^{21,22}$ After $\mathrm{RT}$ magnetic gas sensing using $\mathrm{H}_{2}$ as target gas (Fig. 7b, top) the $\mathrm{O}_{\mathrm{b}}$ contribution remains unchanged, since at RT the formation of $\mathrm{V}_{\mathrm{O}}$ is not favored. ${ }^{7}$ However the relative intensity of $\mathrm{O}_{\mathrm{a}}$ peak is strongly reduced, suggesting that the target gas molecules react preferentially with $\mathrm{O}^{2-}$ ions in the $\mathrm{ZnO}$ crystal, with the consequent formation of a significant amount of surface structural defects, in agreement with the NEXAFS results (Fig. 7a). The $\mathrm{O}_{\mathrm{c}}$ contribution at $532.7 \mathrm{eV}$ establishes because of loosely bound oxygens or $\mathrm{HO}^{-}$species on the ZnO surface, which remain trapped on surface after the reaction.

The $\mathrm{Zn} 2 \mathrm{p}_{3 / 2}$ and $2 \mathrm{p}_{1 / 2}$ peaks are located respectively at 1021.6 and $1044.8 \mathrm{eV}$ (Fig. 7c), with spin-orbit splitting of $23.2 \mathrm{eV} .^{23,24}$ The symmetry of the peaks indicates the presence of a single $\mathrm{Zn}$ specie in the ZnO lattice. No changes on $\mathrm{Zn} 2 \mathrm{p}$ peaks are observed after gas exposure.

\section{Discussion}

The presented device is constituted by a soft ferromagnet and semiconductive piezoelectric $\mathrm{ZnO}$ nanorods (Fig. 2), coupled through the formation of an interface. The latter can be seen as a bi-layer of Co-doped $\mathrm{ZnO}$ and pure $\mathrm{ZnO}$ (Fig. 4). The possibility to magnetically detect gases, also in small concentrations (Fig. 5), can occur thanks to the establishing of a magnetoelastic/-electric coupling at the interface, as also suggested by Sahoo et $a l .{ }^{25}$ and Hsu et $a .^{26}$

Co acts nothing else but as a magnetic transducer and does not react with any target gases, being completely passivated by the formation of the continuous interface layer (Fig. 4). On the contrary, $\mathrm{ZnO}$ is active towards all the tested gases, the highest contribution coming from the nanostructures (Fig. 6 and 7). The reaction with gases only pertains the surface and lateral faces of the NRs, giving rise to an increase in the amount of structural defects (Fig. 7a and b).

From the performed analysis, the magnetic gas sensing mechanism can be described with a two-steps model (Fig. 8). The first step represents the common reaction of the ZnO NRs with reducing gases. ${ }^{27,28}$ Oxygen molecules are surface adsorbed from the environment and trap the free electrons from the surface. Oxygen ions form, which react with the reducing gases. The reaction products are quickly desorbed (Fig. 7) with the concomitant release of free electrons, allowing for a reduction of the $\mathrm{ZnO}$ resistance.

Wurtzite $\mathrm{ZnO}$ crystals are known for being characterized by an electrical polarity and for showing a polarization that can be 


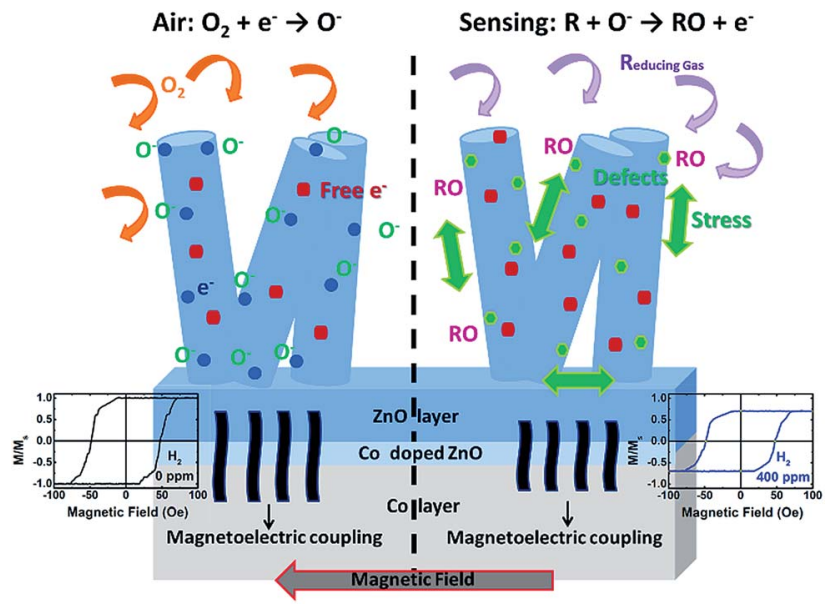

Fig. 8 Schematic representation of the magnetic gas sensing process and an example of the hysteresis loops measured during the sensing process.

either induced by an external strain or spontaneous. ${ }^{29,30}$ The latter is always oriented along the longer axis of the structure and is able to give rise to an electric field which significantly affects the properties of the system. The effect of free charges and ions in changing the spontaneous polarization improves reducing the crystal sizes and is strongly enhanced in nanostructures (Fig. 6). In the second step of the magnetic gas sensing process, the released free electrons redistribute for reducing the electric field and potential created by the spontaneous polarization of the piezoelectric $\mathrm{ZnO}$. This induces a change in the magnetoelectric contribution to the system anisotropy. At the same time, the $\mathrm{V}_{\mathrm{O}}$ and $\mathrm{V}_{\mathrm{Zn}}$ as well as defects (Fig. 7) are responsible for the changes in the surface-ZnO lattice distances. This allows a strengthening of the stress, and the establishing of a magnetoelastic contribution to the system anisotropy. These anisotropy variations are transduced by the Co layer in a decrease of the magnetization, as detected by MOKE measurements (Fig. 5).

It is worth noting that: (i) the MOKE setup, exploited for gas detection can be easily reduced in sizes; (ii) blue or red laser can be used instead of the He-Ne laser, and (iii) the intensity of the applied magnetic field is of only 50 Oe. All these characteristics allow for the implementation of a fast, sensitive, scalable, lowcost and portable magnetic gas sensor, working at room temperature.

\section{E. Conclusions}

In summary, we have successfully developed a new concept class of low-cost magnetic gas sensors, characterized by a high room-temperature stability and sensitivity.

The sensing process involves mainly the ZnO NRs surface and lateral faces, inducing a significant increase of the surfacestructural defectiveness. Co only acts as a magnetic transducer.

The interface between $\mathrm{Co}$ and $\mathrm{ZnO}$ is constituted by a thin Co-doped $\mathrm{ZnO}$ layer whose main role is to reduce the structural and electronic differences between the metal and the semiconductor, allowing for the transduction mechanism of Co. Through an in-depth NEXAFS characterization performed at the $\mathrm{Co}_{2,3}$ edges, we have demonstrated that the Co-doped $\mathrm{ZnO}$ interfacial layer protects the Co layer from oxidation and corrosion during the gas reaction, allowing for the reversibility of the gas sensing process.

The XMCD results demonstrated that this interface does not affect the overall magnetic properties of the system. On the contrary a significant contribution to the magnetization arises from $\mathrm{O}$ vacancies and from the surface and lateral faces of the ZnO NRs.

The formation of structural defects and the change in the $V_{O}$ amount during the reaction dictate a variation of the $\mathrm{ZnO}$ lattice, giving rise to an enhancement of the internal stress. Simultaneously, the piezoelectric properties as well as the polarizability of the ZnO NRs are modified, as a consequence of the free electrons released during the reaction. These phenomena change the magnetoelastic and magnetoelectric contributions to the system anisotropy, transduced by Co in a decrease of the magnetization.

Although this study is fairly fundamental and further investigations are needed, it has the potential to disclose the gate to completely new magnetic gas sensing approaches and achieve a wide range of smart sensing applications for human, health and safety.

\section{Acknowledgements}

We acknowledge the Elettra synchrotron of Trieste that allowed through Proposals No. 20155119 the NEXAFS-XMCD analysis.

\section{References}

1 E. Comini, C. Baratto, G. Faglia, M. Ferroni and G. Sberveglieri, J. Phys. D: Appl. Phys., 2007, 40, 7255.

2 E. Comini, C. Baratto, G. Faglia, M. Ferroni, A. Vomiero and G. Sberveglieri, Prog. Mater. Sci., 2009, 54, 1.

3 A. Punnoose, K. M. Reddy, J. Hays, A. Thurber and M. H. Engelhard, Appl. Phys. Lett., 2006, 89, 112509.

4 Y. G. Sun and H. H. Wang, Adv. Mater., 2007, 19, 2818.

5 A. Punnoose, K. M. Reddy, A. Thurber, J. Hays and M. H. Engelhard, Nanotechnology, 2007, 18, 165502.

6 D. Matatagui, O. V. Kolokoltsev, N. Qureshi, E. V. MejíaUriarte and J. M. Saniger, Nanoscale, 2015, 7, 9607.

7 R. Ciprian, C. Baratto, A. Giglia, K. Koshmak, G. Vinai, M. Donarelli, M. Ferroni, M. Campanini, E. Comini, A. Ponzoni and G. Sberveglieri, RSC Adv., 2016, 6, 42517.

8 P. Sundara Venkatesh and K. Jeganathan, CrystEngComm, 2014, 16, 7426.

9 S. A. Studenikin, N. Golego and M. Cocivera, J. Appl. Phys., 2000, 87, 2413.

10 S. Nannarone, F. Borgatti, A. De Luisa, B. P. Doyle, G. C. Gazzadi, A. Giglia, P. Finetti, N. Mahne, L. Pasquali, M. Pedio, G. Selvaggi, G. Naletto, M. G. Pelizzo and G. Tondello, AIP Conf. Proc., 2004, 704, 450.

11 P. Sundara Venkatesh, C. L. Dong, C. L. Chen, W. F. Pong, K. Asokan and K. Jeganathan, Mater. Lett., 2014, 116, 206. 
12 A. P. Singh, R. Kumar, P. Thakur, N. B. Brookes, K. H. Chae and W. K. Choi, J. Phys.: Condens. Matter, 2009, 21, 185005.

13 H. Thakur, P. Thakur, R. Kumar, N. B. Brookes, K. K. Sharma, A. P. Singh, Y. Kumar, S. Gautam and K. H. Chae, Appl. Phys. Lett., 2011, 98, 192512.

14 P. Thakur, K. H. Chae, J.-Y. Kim, M. Subramanian, R. Jayavel and K. Asokan, Appl. Phys. Lett., 2007, 91, 162503.

15 S. B. Singh, Y.-F. Wang, Y.-C. Shao, H.-Y. Lai, S.-H. Hsieh, M. V. Limaye, C.-H. Chuang, H.-C. Hsueh, H. Wang, J.-W. Chiou, H.-M. Tsai, C.-W. Pao, C.-H. Chen, H.-J. Lin, J.-F. Lee, C.-T. Wu, J.-J. Wu, W.-F. Pong, T. Ohigashi, N. Kosugi, J. Wang, J. Zhou, T. Regierg and T.-K. Sham, Nanoscale, 2014, 6, 9166.

16 I. Lorite, B. Straube, H. Ohldag, P. Kumar, M. Villafuerte, P. Esquinazi, C. E. Rodriguez Torres, S. Perez de Heluani, V. N. Antonov, L. V. Bekenov, A. Ernst, M. Hoffmann, S. K. Nayak, W. A. Adeagbo, G. Fischer and W. Hergert, Appl. Phys. Lett., 2015, 106, 082406.

17 J. W. Chiou, J. C. Jan, H. M. Tsai, C. W. Bao, W. F. Pong, M. H. Tsai, I. H. Hong, R. Klauser, J. F. Lee and J. J. Wu, Appl. Phys. Lett., 2004, 84, 3462.

18 J. W. Chiou, K. P. K. Kumar, J. C. Jan, H. M. Tsai, C. W. Bao, W. F. Pong, F. Z. Chien, M. H. Tsai, I. H. Hong and R. Klauser, Appl. Phys. Lett., 2004, 85, 3220.

19 A. Sharma, A. P. Singh, P. Thakur, N. B. Brookes, S. Kumar, C. G. Lee, R. J. Choudhary, K. D. Verma and R. Kumar, J. Appl. Phys., 2010, 107, 093918.
20 J. W. Chiou, H. M. Tsai, C. W. Pao, K. P. Krishna Kumar, J. H. Chen, D. C. Ling, F. Z. Chien, W. F. Pong, M.-H. Tsai, J. J. Wu, M.-H. Yang, S. C. Liu, I.-H. Hong, C.-H. Chen, H.-J. Lin and J. F. Lee, Appl. Phys. Lett., 2007, 90, 062103.

21 J. Zhao, C. Xie, L. Yang, S. Zhang, G. Zhang and Z. Cai, Appl. Surf. Sci., 2015, 330, 126.

22 J. B. K. Law and J. T. L. Thong, Nanotechnology, 2008, 19, 205502.

23 N. Han, X. Wu, L. Chai, H. Liu and Y. Chen, Sens. Actuators, $B, 2010,150,230$.

24 K. Liu, M. Sakurai and M. Aono, Sens. Actuators, B, 2011, 157, 98.

25 S. Sahoo, S. Polisetty, C.-G. Duan, S. S. Jaswal, E. Y. Tsymbal and C. Binek, Phys. Rev. B: Condens. Matter Mater. Phys., 2007, 76, 092108.

26 C.-C. Hsu, P.-C. Chang, V. R. Muhudinepalli, T.-C. Hsieh, F.-Y. Lo and W.-C. Lin, J. Appl. Phys., 2016, 119, 093905.

27 M. Sinha, R. Mahapatra, B. Mondal, T. Maruyama and R. Ghosh, J. Phys. Chem. C, 2016, 120, 3019.

28 J. J. Hassan, M. A. Mahdi, C. W. Chin, H. Abu-Hassan and Z. Hassan, J. Alloys Compd., 2013, 546, 107.

29 X. Y. Kong and Z. L. Wang, Nano Lett., 2003, 3, 1625.

30 S. Farid, M. Cjoi, D. Datta, M. A. Stroscio and M. Dutta, J. Appl. Phys., 2016, 119, 163108. 